\title{
Growth and Characterisation of Thiourea Doped Sulphamic Acid Single Crystals
}

\author{
B. Kannan ', P. R. Seshadri ${ }^{1 *}$, K. Ilangovan ${ }^{2}$ and P. Murugakoothan ${ }^{3}$ \\ IPG and Research Department of Physics, A.M.Jain College, Meenambakkam, Chennai; galaxycando@gmail.com \\ ${ }^{2} \mathrm{PG}$ and Research Department of Physics, RKM.Vivekananda College, Mylapore, Chennai; seshadri_pr@yahoo.com \\ ${ }^{3} \mathrm{MRDL}, \mathrm{PG}$ and Research Department of Physics, Pachaiyappa's College, Chennai-600030; \\ murugakoothan@yahoo.co.in
}

\begin{abstract}
Thiourea doped Sulphamic acid (Th : SA) single crystals were grown successfully by slow-evaporation technique. To identify the morphology and structure, the as grown crystals were subjected to characterization like powder and single XRD analysis. UVVis-NIR spectrum and FTIR studies are performed to identify the cut-off wavelength and the various functional groups present in the grown crystal. Second harmonic generation were investigated to study the linear and nonlinear optical properties. In order to ascertain the thermal stability of the crystal, thermo-gravimetric analysis (TGA), differential thermo-gravimetric analysis (DTA) and differential scanning calorimetry (DSC) were also carried out.
\end{abstract}

Keywords: NLO, EDAX, Morphology, Optical, FTIR, Thermal.

\section{Introduction}

Sulphamic acid $\left(\mathrm{H}_{2} \mathrm{NSO}_{3} \mathrm{H}\right)$ is an important industrial chemical compound, which is strongly soluble in water. The molecular weight of this compound is 97.09 [1]. Apart from its catalytic and electrometallurgical uses, salts of sulphamic acid have wide applications as anticorrosive agent (or) cross linking agent for polymer [2]. An added advantage in sulphamic acid (SA) is that large single crystals can be grown from low temperature solutions [3]. Hence in the present work, systematic studies on the growth and characterization of thiourea doped sulphamic acid (Th : SA) is reported. The crystalline perfection, structural, presence of funtional groups, thermal, optical behaviours in (Th: SA) are studied by X-ray diffraction, Morphology, FTIR, UV-vis-NIR, TG/DTA, and NLO studies respectively.

*Corresponding author:

P.R. Seshadri (seshadri_pr@yahoo.com)

\section{Experimental Procedure}

The (Th : SA) single crystals are synthesized by dissolving thiourea (Th) and sulphamic acid in the molar ratio 1:1 in double distilled water. (Th: SA) is synthesised according to the relation.

$\mathrm{H}_{2} \mathrm{~N}-\mathrm{CS}-\mathrm{NH}_{2}+\mathrm{HO}-\mathrm{SO}_{2}-\mathrm{NH}_{2} \longrightarrow\left(\mathrm{H}_{2} \mathrm{~N}-\mathrm{CS}-\mathrm{NH}_{3}\right) \mathrm{SO}_{3}-\mathrm{NH}_{2}$ The product is purified by repeated recrystallization, typically thrice from double distilled water. The $100 \mathrm{ml}$ saturated solution is kept in a beaker covered with perforated filter paper at room temperature. The crystals are grown by low temperature solution growth, using solvent evaporation method. In a period of 3 to 4 days, seeds of (Th : SA) single crystals are formed due to spontaneous nucleation. The Figure 1(a) and 1(b) show the photographs of pure SA and (Th : SA) crystals respectively. 


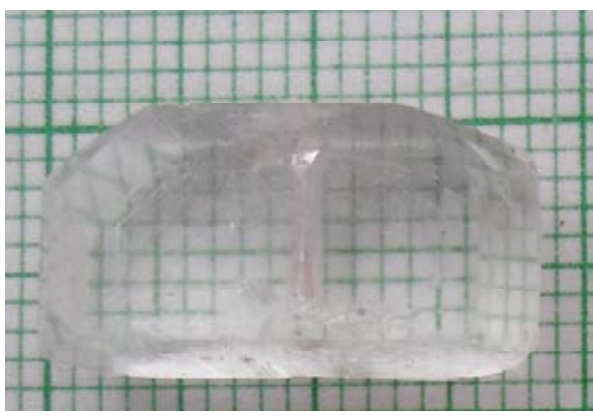

Figure 1(a). Pure Sulphamic acid crystals.

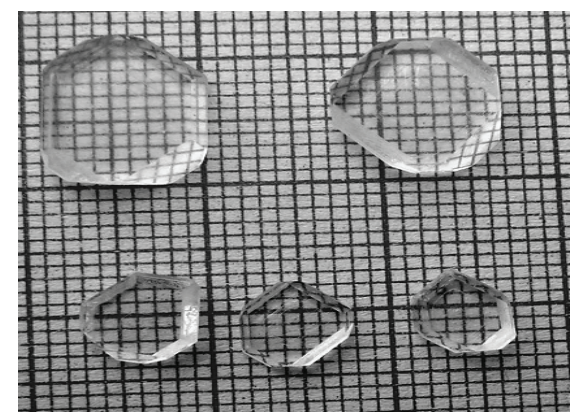

Figure 1(b). Thiourea doped SA crystals.

\section{Results and Discussion}

\subsection{Morphology}

The temperature, concentration, supersaturation, impurities and additives added to the growth solution greatly influence the growth and morphology of the crystals [4]. In the present work the morphological planes of (Th:SA) are located using ENRAF NONIUS CAD-4 diffractometer. The morphology of pure SA and (Th:SA) are shown in Figure 2(a) and 2(b). The (Th : SA) crystal has plate like morphology. The prominent face of (Th: SA) is (100) plane and the other narrow faces are (010), (111), (001) and (201).

\subsection{X-ray Diffraction}

\subsubsection{Single Crystal X-ray Diffraction}

The grown crystal has been subjected to X-ray diffraction studies using ENRAF NONIUS CAD-4 X-ray diffractometer equipped with MoKa radiation $(\lambda=0.7170 \AA \hat{)})$ to determine the unit cell dimensions. The single crystal X-ray diffraction reveals that the crystal belongs to primitive tetragonal system. The cell parameters of SA and (Th : SA) single crystal are tabulated in Table 1. From the obtained data it can be seen that the geometry of the SA crystal changes from lower symmetry to the higher symmetry (ie) from orthorhombic to tetragonal. This may be due to the incorporation of (Th) into the crystal lattice.

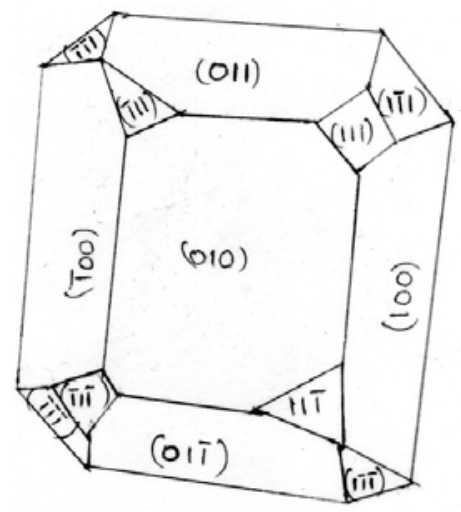

Figure 2(a). Morphology of Pure SA.

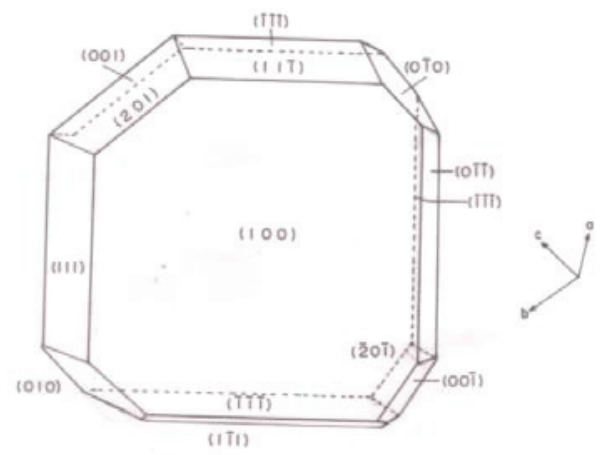

Figure 2(b). Morphology of (Th : SA).

Table 1. Lattice parameters of pure SA and (Th : SA) single crystals

\begin{tabular}{lccc}
\hline Parameters & Pure SA & Th : SA \\
\hline a & $\AA$ & 8.100 & 8.130 \\
b & $\AA$ & 8.049 & 8.130 \\
c & $\AA$ & 9.220 & 9.300 \\
V & $\AA^{3}$ & 604.8 & 614 \\
\hline
\end{tabular}

\subsubsection{Powder X-ray Diffraction}

$\mathrm{X}$-ray powder diffraction pattern of the crystal is recorded on a RICH SEIFERT X-ray diffractometer using $\mathrm{CuKa}(\lambda=$ $1.5406 \AA \hat{)}$ ) radiation. The sample is scanned for a $2 \theta$ range of $10-70^{\circ}$ at a scan rate of $1 \%$ minute. All the observed reflections are indexed. The disclosure of well defined Bragg's peaks at specific $2 \theta$ angle show the high crystallinity of (Th : SA) crystal. The d spacings are calculated using Bragg equation and using the value of $\mathrm{d}$, the hkl values for all the reflections are obtained. The powder X-ray diffraction patterns of pure and doped SA are shown in Figure 3(a) and $3(b)$. 


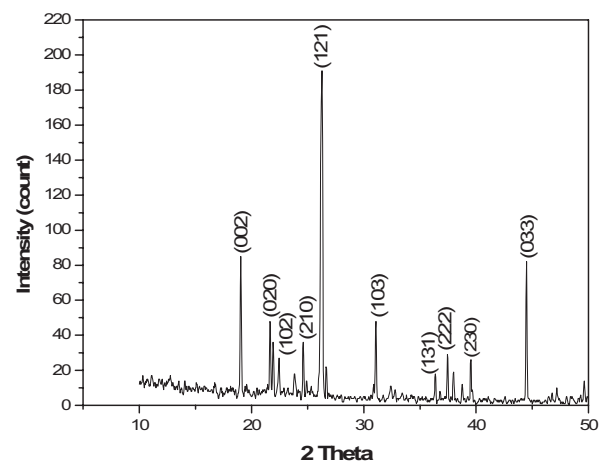

Figure 3(a). Powder XRD of Pure SA.

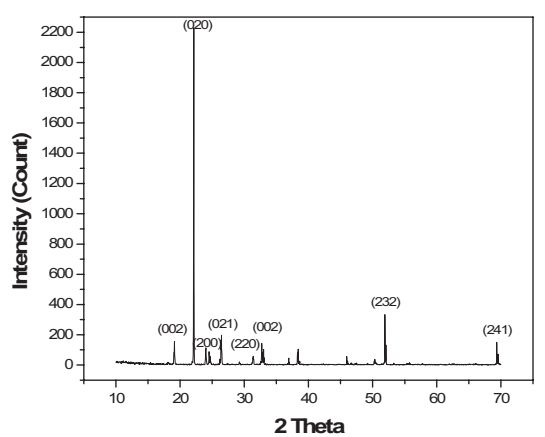

Figure 3(b). Powder XRD of Th : SA.

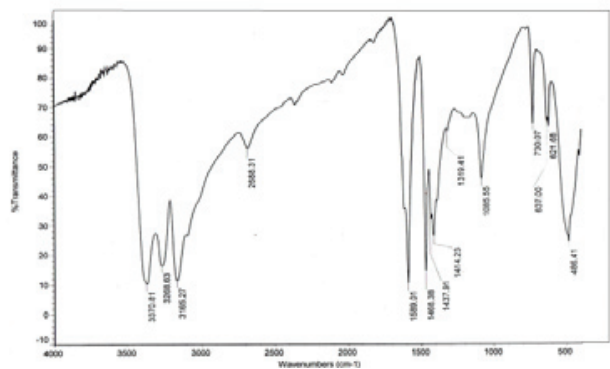

Figure 4. FTIR spectrum of Th : SA.

\subsection{Fourier Transform Infrared Spectroscopy}

The FTIR spectroscopy studies are effectively used to identify the functional groups present in the crystal and to determine the molecular vibrations. The FTIR spectrum for the grown crystal is recorded using BRUKKER IFS $66 \mathrm{v}$ spectrometer by $\mathrm{KBr}$ pellet technique in the range $400-$ $4000 \mathrm{~cm}^{-1}$. FTIR spectrum of (Th: SA) seems to be complex because of various functional groups present in the crystal. The functional group assignments for (Th: SA) is summarized in Table 2.
Table 2. Vibrational band assignment for (Th : SA) single crystal

\begin{tabular}{ll}
\hline Wave number $\left(\mathrm{cm}^{-1}\right)$ & Assignment \\
\hline 3268 & Sym. N-H stretching \\
3370 & Asm N-H stretching \\
2688 & N-S stretching \\
1589 & N-S scissoring \\
1468 & C=S stretching \\
1414 & S=O strecthing \\
1319 & Asm O=SO stretching \\
730 & N-H wagging \\
621 & Sym N-C-N stretching \\
486 & C-N-C bending \\
\hline
\end{tabular}

\subsection{UV-vis-NIR Spectroscopy}

Transmission and absorption spectra are very important for any single crystal which can be useful for any practical application, only if it has a wide transparency window $[5,6]$. In the optical transmission studies, the transmittance of doped crystal has been examined in the wavelength range 200-800 nm using Philips PV8700 UV-visible scanning spectrometer. The recorded absorption spectrum is shown in Figure 5. The UV cut off wavelength of (Th : SA) crystal is found to be at $240 \mathrm{~nm}$ and there is no considerable absorption till $800 \mathrm{~nm}$. It has been observed that the (Th : SA) crystal has increased transparency window compared to the pure SA crystal whose cut off wave length is $270 \mathrm{~nm}$. Hence this crystal can be used for any optical applications.

\subsection{Thermal Studies}

The thermal behavior of the sample is studied using TGA and DTA analysis. The thermogravimetric analysis is carried out between $40^{\circ} \mathrm{C}$ and $1100^{\circ} \mathrm{C}$ at a heating rate of $15^{\circ} \mathrm{C} /$ min in nitrogen atmosphere. The sample is carried out using Perkin-Elmer thermal analysis instrument. Alumina is taken as reference material in $\mathrm{Al}_{2} \mathrm{O}_{3}$ crucible. From the TGA curve it is observed that (Th: SA) exhibits only single stage of decomposition and hence decomposes directly without producing any weight loss dependent of intermediate species. Below $200^{\circ} \mathrm{C}$ there is no detectable weight loss and hence the crystal rejects solvent molecules during crystallization. In the DTA trace at $233.03^{\circ} \mathrm{C}$ explicit endothermic heat effect is observed due to decomposition of the sample and the decomposition extends up to $1000^{\circ} \mathrm{C}$. The high stability of the grown crystal arises due to the strong bonding existing between conjugation layers of sulphamic acid and thiourea [7]. Figure 6. shows the TG / DTA thermograms of (Th : SA). 


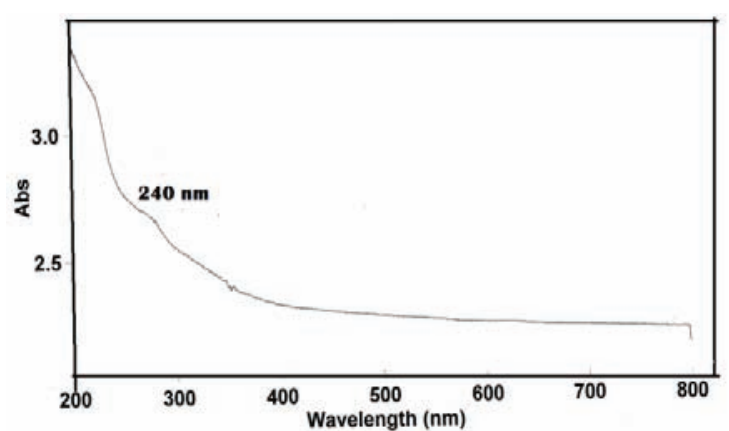

Figure 5. UV-vis-NIR spectrum of (Th : SA) single crystal.

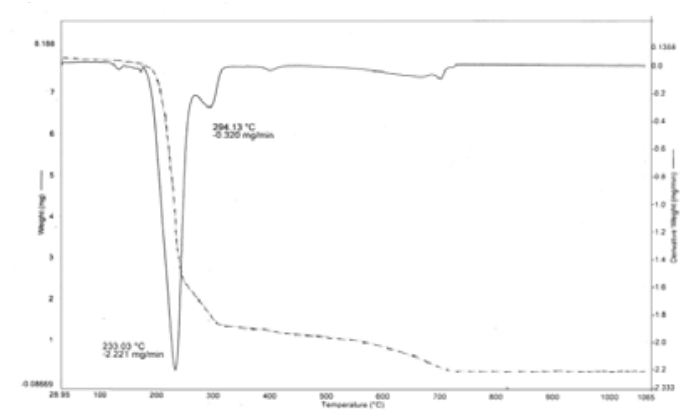

Figure 6. TG / DTA thermograms of (Th : SA).

\subsection{Nonlinear Optical Test}

The NLO materials have many practical applications in research and industries [8-10]. A fundamental beam of wavelength $1064 \mathrm{~nm}$ with a pulse duration of $10 \mathrm{~ns}$ and the frequency repetition of $10 \mathrm{~Hz}$ from Q-switched Nd:YAG laser has been used as a source and passed through the powder sample of particle size $1.229 \mathrm{~nm}$ for second harmonic generation efficiency (SHG) study. Pulse energy and pulse width are maintained as $300 \mathrm{~mJ} \mathrm{~s}^{-1}$ and $10 \mathrm{~ns}$ respectively. The output of (Th: SA) has no green emission similar to pure SA sample. This is interesting, because even though thiourea is an NLO material, it does not induces any NLO property to the grown (Th : SA) crystal. From this study it is observed that the dopant does not induce the NLO property.

\section{Conclusion}

The crystals of (Th : SA) single crystal were grown by slow solvent evaporation technique at room temperature. Using single crystal X-ray diffraction, the unit cell dimensions of (Th : SA) single crystals are determined. Powder XRD studies confirm the crystalline nature of the crystal. The FTIR studies reveal the functional groups of (Th : SA) single crystal. The UV-Vis-NIR absorption spectrum of the crystal indicates the good transmittance in the entire visible region. The thermal studies of (Th : SA) single crystal indicates the high stability of the grown crystal. The non linear optical test reveal that the (Th: SA) single crystal does not shows NLO property.

\section{References}

1. Gharde R A, and Chunarkar D T (2012). Characterization of Sulphamic acid single crystal by optical techniques, International Journal of Scientific and Research Publication, volume 2, issue 6, 1-4.

2. Manickkavachagam R, and Rajaram R K (1984). Crystal structure of anhydrous sodium sulphamate, Zietschrift für Kristallographie, vol 168(1-4), 179-185.

3. Valluvan R, Selvaraju K et al. (2006). Growth and characterisation of sulphamic acid single crystals, Material Chemistry and Physics, vol 97(1), 81-84.

4. Siddheswaran1 R, Sankar R et al. (2006). Nucleation, growth and characterization studies of a nonlinear optical crystal tris allylthiourea, Laser Physics Letters, vol 3(12), 588.

5. Delfinio M, Dougherty J P et al. (1976). Approximate nonlinear optical susceptibility of cubic boracites, Journal of Applied Physics, vol 51(4), 2264-2266.

6. Delfinio M, Loiacono G M et al. (1978). Halide effect in $\mathrm{L}(+)$ glutamic acid halogen acid salts, Journal of solid state Chemistry, vol 23(3-4), 289-296.

7. Marcy H O, Warren L F et al. (1992). Second- harmonic generation in zinc tris(thiourea) sulfate, Applied Optics, vol 31(24), 5051-5060.

8. Kalaiselvan S, Pasupathi G et al. (2012). Growth and characterization of a new metal-organic crystal: Sodium thiourea bromide, Der Pharma Chemica, 2012, vol 4(5), 1826-1832.

9. Kannan B, Seshadri P R et al. (2013). Growth and characterization of lanthanum doped sulphamic acid, Indian Journal of Science and Technology, vol 6(4), 4357-4361.

10. Kannan B, Seshadri P R et al. (2013). Growth and characterization of Cerium doped Sulphamic acid, Asian Journal of Chemistry, vol 25(12), (Accepted). 